\title{
Repair of a condylar fracture with a titanium miniplate via two approaches
}

\author{
Abstract \\ Objective: To present a safe and reliable technique for open reduction with internal fixation \\ for condylar neck fractures. \\ Methods: Design: Case Report \\ Setting: Tertiary Government Training Hospital \\ Patient: One \\ Results: A 25-year-old male patient with a unilateral condylar neck fracture presented with \\ gross deformity and malocclusion. The patient underwent an open reduction with internal \\ fixation using a 16-hole titanium plate via a preauricular and submandibular incision. The \\ surgical outcome provided the patient with a restored vertical dimension of the mandibular \\ ramus, a good occlusion, acceptable scars, an intact Facial nerve, a stable fixation, and an \\ early mobilization of the temporomandibular joint.
}

Conclusion: Using a single 16-hole titanium miniplate for condylar neck fractures through two approaches resulted in the avoidance of exposure and trauma to the facial nerve and its branches. Replication of the technique by any surgeon would still result to the same outcome: a short shallow learning curve, decreased operative time, a safe Facial nerve, and a satisfied patient.

Keywords: mandibular fracture, condylar fracture, condylar neck, open reduction with internal fixation, miniplate osteosynthesis, titanium, miniplate, transparotid approach, preauricular approach, submandibular approach, minimally invasive surgery, endoscopic surgery of mandible, facial nerve, temporomandibular joint
Volume 10 Issue 3 - 2018

\author{
Rene C Lacanilao, Avian Loren C Carlos \\ Department of Otolaryngology-Head \& Neck Surgery,Amang \\ Rodriguez Memorial Medical Center, Philippines
}

\author{
Correspondence: Rene C. Lacanilao, Department of \\ Otolaryngology-Head \& Neck Surgery, Amang Rodriguez \\ Memorial Medical Center, Department of Health, Sumulong \\ Hi-way, Marikina City, MetroManila, Philippines \\ Emailarmmc_orlhns@yahoo.com
}

Received: December 17, 2017| Published: June 26, 2018

\section{Introduction}

One of the major complications in the repair of a mandibular condylar neck fracture is injury to the Facial nerve and its branches. Techniques vary. More recent studies recommend the use of an open technique with internal fixation since it allows the surgeon to directly approximate the fragments and results in better outcomes for the patient.,3

The controversies of using an open surgical technique versus a closed technique with MaxilloMandibular Fixation (MMF) still exist between different surgeons. On one side are surgeons who are for the reduction and fixation of all completely displaced condylar fractures. On the other side are surgeons who choose not to open the condyle because they were poorly trained, not versed with the technique, or are afraid of the approach to the temporomandibular joint and condyle so opt to apply conservative approaches even for medially displaced condylar heads.

Open procedures to the condyle require a long surgical time of 3-5 hours. Aside from this, complications for the existing open reduction procedures, even with minimally invasive techniques through endoscopic surgery, include facial palsy, secondary infection, fistula formation, and scarring..$^{1,2}$ Although conservative techniques like MMF avoided these it doesn't mean that complications doesn't exist. Patients treated with conservative techniques have persistently poor occlusion and chewing problems, temporomandibular joint pains and arthalgia.

The study aims to present a safe and reliable surgical technique for the open reduction of mandibular condylar neck fractures using a single 16-hole titanium miniplate.

\section{Case report}

A 25-year old, male, patient presented with a comminuted mandibular fracture of the following areas: an Alveolar Fracture, Left; Parasymphyseal Fracture, Left; and a Condylar Neck Fracture, Right as shown in Figure 1. The patient complained of a gross deformity with malocclusion (Figure 2).



Figure I Pre-operative Panoramic X-ray showing a displaced right condyle on the left side of the picture.

\section{Surgical technique}

The condylar neck fracture was identified through the preauricular incision and the proximal segment was verified to be displaced medially. A submandibular incision was used to expose the angle of the right hemimandible. A subperiosteal tunnel from the superior incision to the inferior incision was created using a Freer. By sliding 
the Freer subperiosteally, the Facial nerve was safely avoided (Figure 3) (Figure 4). After the condylar head was identified and returned to the empty glenoid fossa the fragment was rotated then held and oriented by means of a $7 \mathrm{~mm}$. titanium self-tapping screw that was screwed half-way into the condylar segment that served as its handle and stabilizer. The angle of the right hemimandible was then grasped using a bone holder and downward traction was applied to make space to facilitate repositioning the condylar fragment. MMF and Gilmer wires have not been applied. This was to facilitate traction of the mandible downwards.

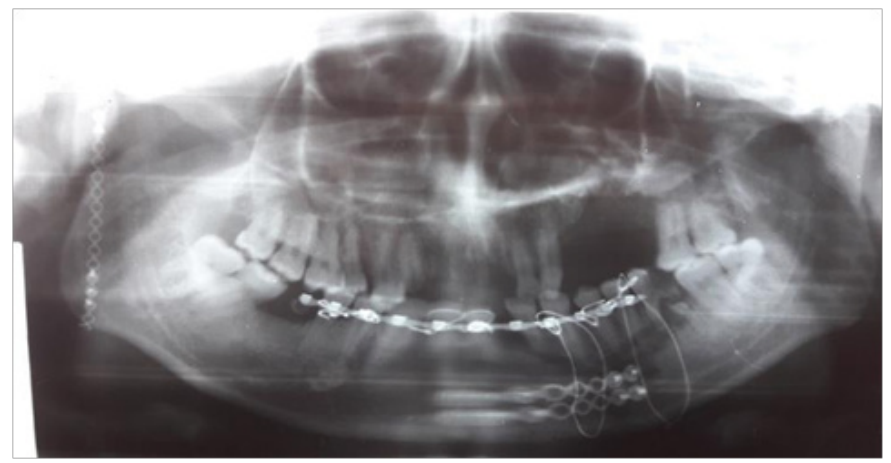

Figure 2 Post-operative Panoramic X-ray.The miniplate lies over the realigned condyle on the left.

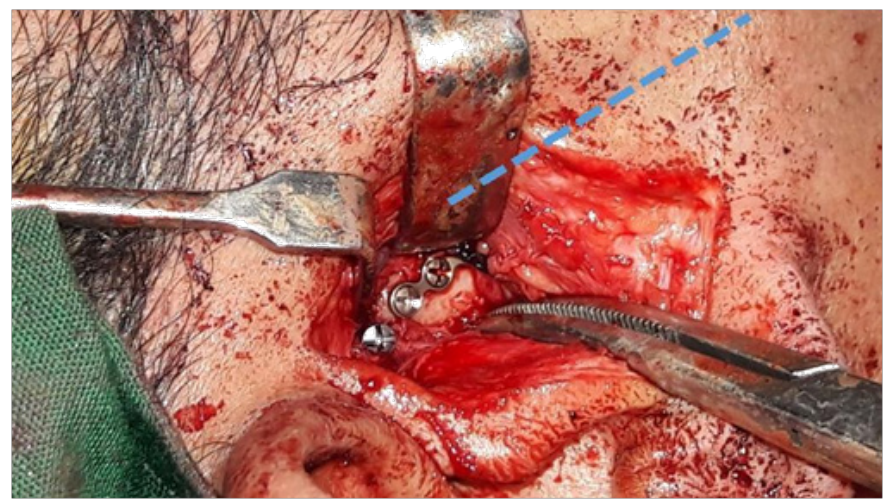

Figure 3 Placement of long screw and Proximal End of the Titanium Plate on the Proximal Segment of the Condylar Neck Fracture (dotted lines show the lie of the miniplate).

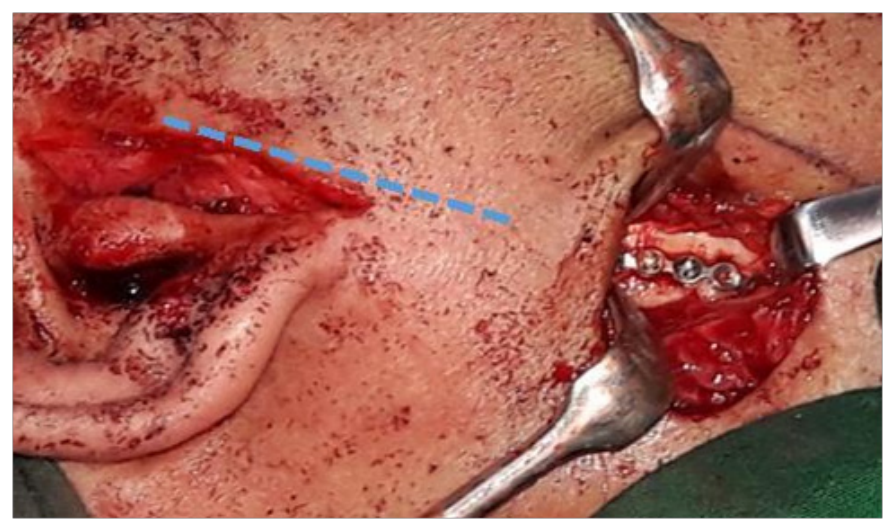

Figure 4 Distal End of the Titanium Plate fixed to the Ramus of the Mandible (dotted lines show the lie of the miniplate).
A 16-hole $1.5 \mathrm{~mm}$ semi-rigid, miniplate was slid through the tunnel and fixed to the condylar fragment with two screws. The caudal portion of the titanium plate was then used to fine tune the distance and orientation of the condylar fragment. The lie was verified visually and by palpation using the end of the Freer by feeling for the bones. Three screws were then fixed on the bottom of the plate near the angle of the mandible. The excess miniplate was molded to the edge of the angle of the mandible (Figure 5).

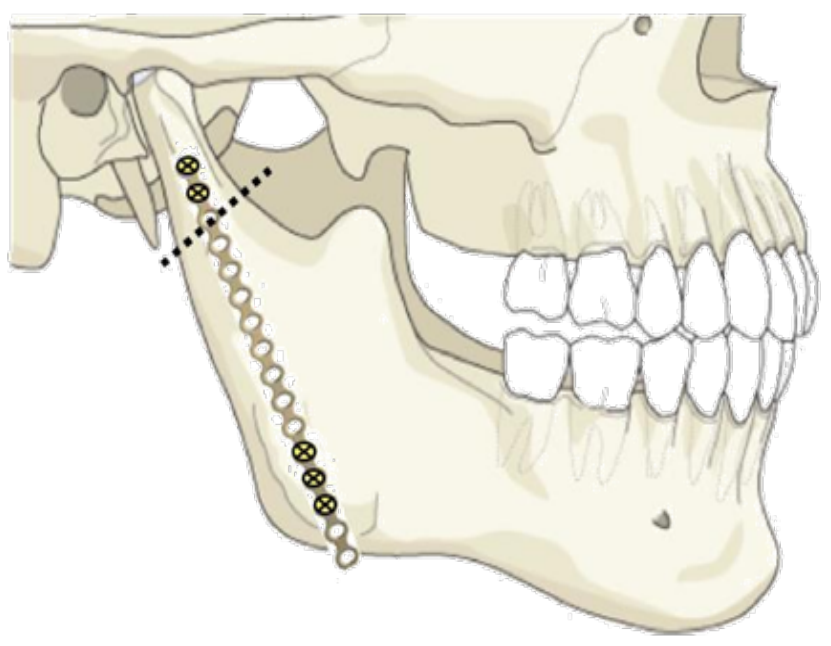

Figure 5 A Diagrammatic Representation of the Condylar Neck Fracture (dotted lines) with the length of the Miniplate Applied and the placement of the screws.

The rest of the fractures were reduced and fixed using standard techniques. The incision sites were then closed by layers. MMF was not maintained post operatively. The patient was maintained on NGT feedings for ten days after which a soft diet was given. Passive mouth opening exercises were also instituted.

\section{Outcome}

Post-operatively, the patient was asked for his occlusion status and the patient reported it to be excellent. There was no trismus. The patient was able to open and close his mouth without difficulty. The patient had fine linear scars hidden from direct view.

Use of a long 16-hole titanium miniplate in this type of fracture resulted in the avoidance of exposure and trauma to the facial nerve and its branches. The patient never presented with a transient nor a delayed facial nerve paralysis. There were no reports of hematoma formation on the preauricular area throughout the course in the ward.

The post-operative panoramic x-ray. Showed the occlusion and the realignment of the condylar neck fracture on the right with the long titanium plate in place. The vertical dimension of the right mandibular ramus very nearly approximates the height of the left side .

\section{Discussion}

For a long time the technique favored for condylar fractures has been closed reduction with MaxilloMandibular Fixation because the alternative of opening the temporomandibular joint was difficult, fraught with complications, fixation with wiring was hard to apply, and the results unpredictable. Newer techniques with the use of compression plates, Delta plates, 3D plates, 4 hole miniplates, pins, 
and total mandibular condylar replacements were developed and the possibility of improved condylar fracture repair became possible.

At the present time open reduction with internal fixation (ORIF) is a favored technique for displaced mandibular condylar neck fractures despite its risks. ${ }^{1}$ This is due to advances in surgical techniques, materials used, and better imaging modalities. Still, a major complication in the repair of a mandibular condylar neck fracture is injury to the Facial nerve (Figure 6). Using the technique described in this case report enables the surgeon to directly reduce and fix the displaced condylar neck fracture while minimizing injury to the Facial nerve. There was no need to locate or identify the Facial nerve and the surgeon could proceed directly to creating the two incisions that would be needed to reduce and fixate the fractured segments. The method of foregoing Facial nerve identification in condylar repair was also mentioned by Haug, et al. ${ }^{3}$ The study reported the use of minimally invasive endoscopic techniques to fix the mandibular condylar fracture. However, using Haug's technique required a steep learning curve. ${ }^{4}$ The longest operative time was reported to be at 4 hours and 40 minutes and despite the use of endoscopes facial palsies were still reported. As a result, the use of endoscopy has not been universally recommended for condylar neck fractures due to the prolonged operative time, scar perception, and facial nerve palsy. Because of this endoscopic repair of condylar fractures has not gained worldwide acceptance.

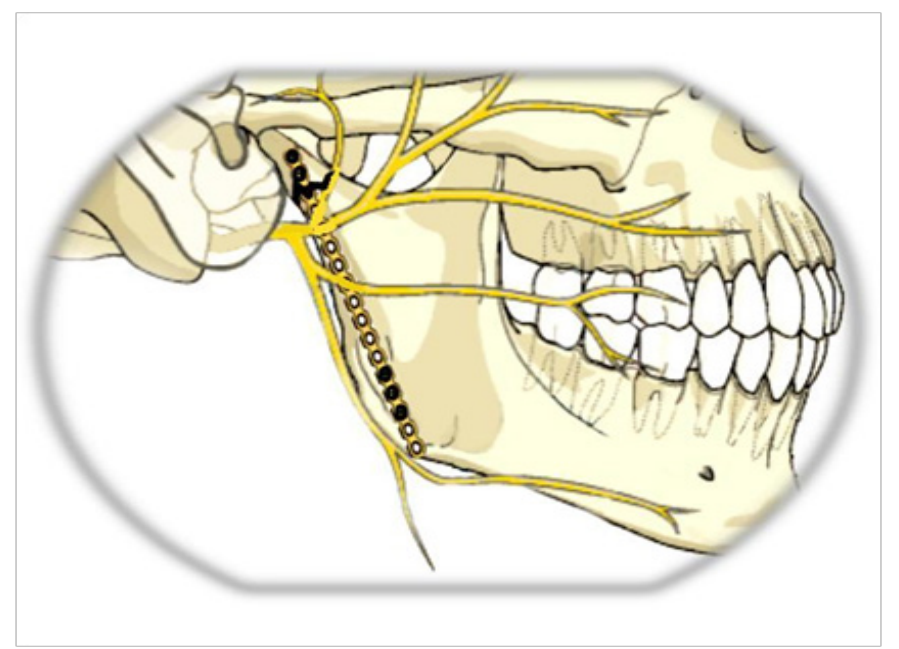

Figure 6 A Diagrammatic Representation of the position of the miniplate as it lies under the Facial nerve.

With regards to the skin incisions several studies presented the different incisions used in approaching the temporomandibular joint and condyle. Spinzia, et al., ${ }^{1}$ recognized several approaches including the preauricular, retroauricular, submandibular, coronal, intraoral or a combination of these approaches. Since there is still no universally accepted standard procedure for fixing a mandibular condyle fracture, different approaches can be utilized to repair condylar fractures. The principle of staying beneath the superficial layer of the temporal fascia provides for a safe facial nerve. ${ }^{1}$ The benefits of having a safe facial nerve outweighs the risk of having surgical scars. These scars are not much of an issue if the preauricular incision is created adequately small for a direct access to the condyle and the submandibular incision is employed through the natural skin crease. ${ }^{5}$
Open reduction using a long titanium plate fixed with two screws to the condylar fragment and three screws distally near the angle is a safe and an easy technique for any surgeon. ${ }^{6}$ Creating a preauricular incision and sliding the extended titanium plate through the mandibular periosteum, beneath the superficial layer of the temporalis fascia avoids injury to the Facial nerve. Applying a 16-hole miniplate provides a stable fixation of the condylar neck fracture. Incisions created at the natural skin creases provides acceptable cosmesis. ${ }^{7,8}$ Overall, it resulted with the patient having a restored vertical dimension of the mandibular ramus, a good occlusion, acceptable scars, an intact facial nerve, a stable fixation, and an early mobilization for the temporomandibular joint. The authors are convinced that when any surgeon applies this same simple technique, the outcome will be the same: a short learning curve, a decreased operative time, a safe Facial nerve, and a satisfied patient with a return to his premorbid occlusion.

\section{Conclusion}

This Surgical Technique offers the surgeon a safe, fast, effective and reliable reduction and fixation of the fractured condyle. The displaced condyle can be directly reduced back into its position and plated while avoiding the Facial Nerve. The technique is promising. It may not be perfect and improvements can be made but it is simple, can be replicated, has a minimal learning curve produces a surgery with a shorter operative time, a satisfied patient with a near to premorbid occlusion and early joint mobilization.

\section{Acknowledgments}

None.

\section{Conflict of interest}

The author declares there is no conflict of interest.

\section{References}

1. Spinzia, A, Patrone R, Belli E, et al. Open reduction and internal fixation of extracapsular mandibular condyle fractures: A long-term clinical and radiological follow-up of 25 patients. BMC Surg. 2014;14:68.

2. Choi KY, Yang JD, Chung HY, et al. Current concepts in the Mandibular Condyle fracture management part II: Open reduction versus closed reduction. Arch Plast Surg. 2012;39(4):301-8.

3. Haug RH, Brandt MT. Closed Reduction, Open Reduction, and Endoscopic AssistanceL Current Thoughts on the Management of Mandibular Condyle Fractures. Plastic and Reconstructive Surgery. 120(7):90S-102S.

4. Schoen R, Fakler O, Metzeger MC, et al. Preliminary functional results of endoscope-assisted transoral treatment of displaced bilateral condylar mandible fractures. Int J Oral Maxillofac Surg. 2008;37:111-116.

5. Booth P, Eppley B, Schmelzeisen R. Maxillofacial trauma and esthetic facial reconstruction. Spain: Elsevier Science; 2003.

6. Elgazzar R. Open versus Closed Treatment of Mandibular Condyle Fracture. Dentistry. 2012; 02(07).

7. Mathog R, Carron M, Shibuya T. Mathog's Atlas of Craniofacial Trauma. 2nd ed. Philadelphia: Wolters Kluwer Health/Lipincott Williams \& Wilkins; 2012.

8. Oliver R. Condylar fractures: Is open or closed reduction best? Evid Based Dent. 2008;9(3):84-84. 